\title{
Positive Foliations on Compact Complex Manifolds
}

\author{
Edoardo BALLICO
}

University of Trento

(Communicated by R. Miyaoka)

\section{Introduction}

We recall the following definition of singular meromorphic foliation.

Definition 1.1. Let $X$ be a smooth complex compact $n$-dimensional manifold. A dimension $q, 1 \leq q<n$, singular meromorphic foliation on $X$ is defined by a rank $q$ subsheaf $E$ of the tangent bundle $T X$ of $X$ such that $T X / E$ has no torsion and $E$ is involutive, i.e. $E$ is closed under Lie brackets.

If $q=1$ (i.e. if the foliation is a foliation by curves) then the condition of involutiveness is automatically satisfied and $\mathrm{E}$ is a line bundle ([H], Prop. 1.9). The sheaf $E$ is called the tangent sheaf to the leaves of the foliation. In this paper we study positive singular meromorphic foliations, mainly in the case in which $X$ has a fibration.

DEFINITION 1.2. Let $X$ be a smooth complex compact $n$-dimensional manifold and $F$ a dimension $q, 1 \leq q<n$, singular meromorphic foliation on $X$ defined by an exact sequence

$$
0 \rightarrow E \rightarrow T X \rightarrow T X / E \rightarrow 0
$$

with $E$ involutive, $\operatorname{rank}(E)=q$, and $T X / E$ torsion free.

(a) We will say that $F$ is effective or non-negative if the coherent sheaf $E$ is generically spanned by its global sections, i.e. there is a non-empty open subset $U$ of $X$ such that the natural map $H^{0}(X, E) \otimes \boldsymbol{O}_{X} \rightarrow E$ is surjective at every point of $U$.

(b) We will say that $F$ is semi-positive if we may take as $U$ a Zariski open subset of $X$ such that $X \backslash U$ is a closed analytic subset of $X$ with codimension at least two.

(c) Assume $q=1$. Then we will say that $F$ is strictly generically positive if $h^{0}(X, E) \geq$ 2 and the base locus of $E$ has codimension at least two in $X$ and we will say that $E$ is strictly generically effective if $h^{0}(X, E) \geq 2$.

We need to use some kind of positivity for torsion-free coherent sheaves on compact complex manifolds.

Received July 3, 2001; revised June 3, 2002 
DEFINITION 1.3. Let $X$ be a connected compact complex manifold and $E$ a torsionfree coherent sheaf on $X$. We will say that $E$ is strictly generically positive if there is an open subset $U$ of $X$ such that $X \backslash U$ is an analytic subset of codimension at least two in $X$ and for every connected compact complex manifold $W$ and every non constant holomorphic map $f: W \rightarrow X$ with $f(W) \cap U \neq \emptyset$ we have $h^{0}\left(W, \operatorname{Hom}\left(f^{*}(E), \boldsymbol{O}_{W}\right)\right)=0$.

Inspired by [G], Def. 2.5, and [B] we introduce the following definition.

Definition 1.4. Let $f: X \rightarrow B$ be a holomorphic map between smooth complex compact manifolds such that the general fiber of $f$ is a smooth connected curve. Let $F$ be a singular meromorphic foliation by curves on $X$ associated to a non-zero section of $T X \otimes$ $L, L \in \operatorname{Pic}(X)$, i.e. induced by a non-zero map $u: L^{*} \rightarrow T X$ such that sheaf $T X / u\left(L^{*}\right)$ is locally free outside a codimension $\geq 2$ subvariety of $X$. We will say that $F$ is a semi-Riccati foliation if there are $R \in \operatorname{Pic}(B)$ and a divisor $D$ on $\mathrm{X}$ with $D \geq 0$ and $\operatorname{dim}(f(D))<\operatorname{dim}(D)$ such that $L^{*} \cong f^{*}(R)(D)$.

For any smooth connected compact complex manifold $X$, let $\operatorname{Alb}(X)$ be its Albanese torus and (up to a choice of a point of $X$ ) let $\alpha: X \rightarrow \operatorname{Alb}(X)$ be the Albanese mapping of $X$ ([U1], Def. 9.6, or [U2]).

In section two we will prove the following results.

THEOREM 1.5. Let $X$ be a connected compact complex manifold with $\operatorname{dim}(X)=3$ and Kodaira dimension $\kappa(X)=0$. Let $\alpha: X \rightarrow \operatorname{Alb}(X)$ be the Albanese mapping of $X$. Assume $\operatorname{dim}(\alpha(X))=1$. Let $F$ be a singular effective meromorphic foliation by curves on $X$. Assume that $F$ is not everywhere transversal to a general fiber of $\alpha$. Then we get the following:

(1) The foliation $F$ is semi-Riccati and it induces a regular meromorphic foliation by curves on every smooth fiber of $\alpha$.

(2) Every smooth fiber of $\alpha$ is a smooth connected minimal complex surface isomorphic either to a torus or to a primary Kodaira surface. Furthermore, we get the following:

(2-1) If a smooth fiber, $Y$, of $\alpha$ is a torus, then the corresponding foliation on $Y$ is induced by a linear foliation on the universal covering $C^{2}$ of $Y$.

(2-2) If a smooth fiber, $Y$, of $\alpha$ is a primary Kodaira surface, then $\alpha(X)$ is a smooth elliptic curve and the corresponding foliation on $Y$ is induced by a submersion $m: Y \rightarrow B$ with $B$ elliptic curve, each fiber of $m$ being isomorphic to an elliptic curve. Furthermore, $F$ is not strictly generically effective.

THEOREM 1.6. Let $f: X \rightarrow B$ be a surjective holomorphic map between smooth connected complex manifolds with $\operatorname{dim}(X)>\operatorname{dim}(B)>0$. Set $m:=\operatorname{dim}(X)-\operatorname{dim}(B)$. Let $G^{\prime}$ be a general fiber of $f$ and $G$ any connected component of $G^{\prime}$. Fix an integer $q$ with $1 \leq q<\operatorname{dim}(X)$ and assume the existence of a dimension $q$ generically strictly positive singular foliation $F$ on $X$. Then we get the following:

(1) $q \leq m$. 
(2) If $q=m$, then $F$ is induced by the fibration $f$ and the tangent bundle $T G$ of $G$ is strictly generically positive.

(3) If $q<m$, then $F$ induces a strictly positive dimension $q$ singular meromorphic foliation on $G$.

The statement of Theorem 1.6 says that any strictly generically positive foliation on a smooth compact complex manifold $X$ commutes with any fibration on $X$.

We will be particularly interested in the case in which $X$ is not algebraic. The field $\mathbf{C}(X)$ of all meromorphic functions on $X$ is a finitely generated extension of the field $\mathbf{C}$ and its trascendence degree, $a(X)$, over $\mathbf{C}$ is called the algebraic dimension of $X$; we have $0 \leq a(X) \leq n$ and $a(X)=n$ if and only if $X$ is Moishezon, i.e. bimeromorphic to a compact algebraic smooth variety ([U1], pp. 24-27). In section two we will prove the following result.

THEOREM 1.7. Let $X$ be a smooth compact complex $n$-dimensional manifold with $a(X)=n-1$. There is a compact manifold $W$, a bimeromorphic morphism $u: W \rightarrow X$ and a morphism $f: W \rightarrow B$ such that $B$ is a smooth $(n-1)$-dimensional projective variety and a general fiber of $f$ is an elliptic curve. Every singular meromorphic foliation on $X$ induces a singular meromorphic foliation on $W$. Every singular meromorphic foliation by curves on $W$ is semi-Riccati with respect to the morphism $f: W \rightarrow B$.

We were motivated from [G], [B], [U1] and [U2]. The papers [G] and [B] made clear that semi-Riccati foliations and fibered surfaces are important in the theory of foliations on surfaces. In the classification of non-algebraic compact complex manifolds two very natural fibrations occur: the algebraic reduction and the Albanese mapping. It seemed to us important to extend to the $n$-dimensional case the interplay between the existence of singular foliations and the existence of fibrations. This paper contains the best we could do on this topic. We do not know any paper considering it from this point of view.

This research was partially supported by MURST and GNSAGA of INdAM (Italy).

\section{The proofs}

REMARK 2.1. Let $X$ be a smooth complex compact non-rational surface, $\mathrm{Y}$ its minimal model and $f: X \rightarrow Y$ its associated morphism. Hence there is a finite set $S \subset Y$ such that $f$ induces an isomorphism $\phi$ of $f^{-1}(Y \backslash S)$ onto $Y \backslash S$. For every singular meromorphic foliation $F$ on $X$ there is a unique singular meromorphic foliation $F^{\prime}$ on $Y$ such that $F^{\prime} \mid(Y \backslash S)=\phi f^{-1 *}\left(F \mid\left(f^{-1}(Y \backslash S)\right)\right.$.

REMARK 2.2. Let $f: X \rightarrow B$ be a holomorphic map between smooth complex compact manifolds such that the general fiber of $f$ is a smooth connected curve of genus $g$ and $H$ the singular meromorphic foliation on $X$ associate to the fibration $f$. The singular meromorphic foliation $H$ is semi-Riccati if and only if $g=1$. If $\operatorname{dim}(X)=2$ and $f: X \rightarrow B$ is a fibering by elliptic curves, then a turbulent foliation in the sense of [B], p. 581, is semiRiccati. 
PROOF OF 1.7. By [U1], Th. 12.4, there is a compact complex manifold $W$, a bimeromorphic morphism $u: W \rightarrow X$ and a morphism $f: W \rightarrow B$ such that $B$ is a smooth $(n-1)$-dimensional projective variety and a general fiber of $f$ is a smooth elliptic curve. Any singular meromorphic foliation by curves on $X$ induces a singular meromorphic foliation by curves on $W$. Take any singular meromorphic foliation by curves $F$ on $W$. By [H], Prop. 1.9, there is $L \in \operatorname{Pic}(W)$ and $\sigma \in H^{0}(W, T W \otimes L) \backslash\{0\}$ such that the zero-locus of $\sigma$ contains no hypersurface of $W$. Let $G$ be a general fiber of $f$.

Claim. For every $R \in \operatorname{Pic}(W)$ we have $\operatorname{deg}(R \mid G)=0$.

Proof of the Claim. Assume the existence of $R \in \operatorname{Pic}(W)$ with $\operatorname{deg}(R \mid G) \neq 0$. Taking $R^{*}$ instead of $R$ if necessary, we may assume $\operatorname{deg}(R \mid G)>0$. Set $\gamma:=\operatorname{deg}(R \mid G)$. Taking $R^{\otimes 2}$ instead of $R$ if necessary, we may assume $\gamma \geq 2$. Take $H \in \operatorname{Pic}(B)$ with $H$ very ample. Set $L_{m}:=R+m f^{*}(H)$. Since $\operatorname{deg}\left(L_{m} \mid G\right)=\gamma>0$, we have $H^{i}\left(G, L_{m} \mid G\right)=0$ for every $i>0$ and $h^{0}\left(G, L_{m} \mid G\right)=\delta$ (Riemann-Roch). Thus for every integer $i>0$ the coherent sheaf $R^{i} f_{*}\left(L_{m}\right)$ is supported by a proper analytic subspace of $B$, while $R^{0} f_{*}\left(L_{m}\right)$ is a torsion free rank $\gamma$ sheaf on $B$. By the projection formula and the ampleness of $H$, for all integers $m \gg 0$ the sheaf $R^{0} f_{*}\left(L_{m}\right)$ is spanned by its global sections. Hence we obtain the existence of a non-zero section $s$ of $L_{m}, m \gg 0$. Call $T$ the effective Cartier divisor of $W$ associated to $s$. By the generality of $G$ the section $s \mid G$ of $L_{m} \mid G$ is not identically zero. Since $\operatorname{deg}\left(L_{m} \mid G\right)=\gamma, G \cap T$ is a zero-dimensional scheme of length $\delta$. Hence $T$ is a hypersurface of $W$ with $f(T)=B$ and $\operatorname{deg}(f \mid T)=\gamma \geq 2$, contradicting a property of the algebraic reduction of $X$ (use either [U1], Th. 12.1, or [U1], condition 3) in Definition 3.3) and proving the claim.

Since $G$ is a smooth fiber of $f$, the normal bundle $N_{G / W}$ of $G$ in $W$ is isomorphic to $\boldsymbol{O}_{G}^{\oplus(n-1)}$. Since $G$ is an elliptic curve, $T G \cong \boldsymbol{O}_{G}$. Since $T W \mid G$ is an extension of $N_{G / W}$ by $T G, T W \mid G$ is an extension of $\boldsymbol{O}_{G}^{\oplus(n-1)}$ by $\boldsymbol{O}_{G}$. By the generality of $G$ the section $s$ induces a non-zero section of $(T W \otimes L) \mid G$. We just saw that $(T W \otimes L) \mid G$ is an extension of $(L \mid G)^{\oplus(n-1)}$ by $L \mid G$. Thus $0<h^{0}(G,(T W \otimes L) \mid G) \leq n\left(h^{0}(G, L \mid G)\right)$. By the Claim we have $\operatorname{deg}(L \mid G)=0$. Since a degree zero line bundle on $G$ has a non-zero section if and only if it is trivial, we have $L \mid G \cong \boldsymbol{O}_{G}$. Thus $L^{*} \mid G \cong \boldsymbol{O}_{G}$. Hence $R^{0} f_{*}\left(L^{*}\right)$ is a rank 1 torsion free sheaf on $B$. Since $B$ is smooth, the rank 1 torsion free sheaf $A:=\left(R^{0} f_{*}\left(L^{*}\right)\right)^{* *}$ is a line bundle ([H], Prop. 1.9). By a theorem of base change ([MS], Prop. 4 and Cor. 2, and [OSS], p. 11) the natural map $\delta: f^{*}\left(R^{0} f_{*}\left(L^{*}\right)\right) \rightarrow L^{*}$ is injective over $G$. Hence $\delta$ induces a natural non-zero map $f^{*}(A) \rightarrow L^{*}$ whose restriction to a general fiber of $f$ is an isomorphism. Hence the line bundle $L^{*} \otimes f^{*}(A)^{*}$ is associated to a Cartier divisor $D$ with $D \geq 0$ and $\operatorname{dim}(f(D)) \leq n-2$. Thus $F$ is a semi-Riccati foliation.

REMARK 2.3. Take $q=1$ in Definition 1.2, i.e. consider a singular meromorphic foliation by curves on $X$. Hence $\operatorname{rank}(E)=1$. By [H], Prop. 1.9, $E$ is a line bundle on $X$. It is called the tangent bundle to the foliation $F$. The foliation $F$ is non-negative if and only 
if there is a Cartier divisor $D \geq 0$ such that $E \cong \boldsymbol{O}_{X}(D)$. The foliation $F$ is semi-positive if and only if either $D=0$ or the linear system $|D|$ has no base component. The foliation $F$ is strictly generically positive if and only if $D \neq 0$ and the linear system $|D|$ has no base component, while it is strictly generically effective if there is a Cartier divisor $A \geq 0$ such that $D(-A) \geq 0$ and the rational map induced by $|D(-A)|$ is not constant and defined outside a codimension two subspace of $X$.

REMARK 2.4. Let $X$ be a smooth complex compact manifold with $h^{0}(X, T X)=0$. Then $X$ has no effective singular meromorphic foliation by curves.

REMARK 2.5. Let $X$ be a smooth complex compact surface and $Y$ its minimal model. If $h^{0}(X, T X) \neq 0$, then $h^{0}(Y, T Y) \neq 0$. Look at Table 10 at page 188 of [BPV] with a list of all minimal smooth surfaces with Kodaira dimension 0 . If $Y$ is a hyperelliptic surface, then $h^{0}(Y, T Y)=0$. If $Y$ is a $K 3$ surface, then $h^{0}(Y, T Y)=0$ because $K_{Y}$ trivial implies $T Y \cong \Omega_{Y}$ and $h^{0}\left(Y, \Omega_{Y}\right) \leq h^{1}\left(Y, \boldsymbol{O}_{Y}\right)=0$. If $Y$ is an Enriques surface, then it has a $K 3$ surface as unramified double covering and hence $h^{0}(Y, T Y)=0$. Now assume that $Y$ is a primary Kodaira surface ([BPV], Ch. V, Sect. 5). Thus $K_{Y} \cong \boldsymbol{O}_{Y}$, there is an elliptic curve $C \subset Y$ and a submersion $m: Y \rightarrow \mathbf{P}^{1}$ such that every fiber of $m$ is isomorphic to $C$. We have $h^{0}(Y, T Y)=h^{0}\left(Y, \Omega_{Y}\right)=h^{1}\left(Y, \boldsymbol{O}_{Y}\right)=1$ and hence there is a unique effective foliation on $Y$. This foliation is associated to the submersion $m$ and hence it is regular. A secondary Kodaira surfaces has no regular holomorphic vector field.

REMARK 2.6. Let $Y$ be an $n$-dimensional complex torus and $F$ a dimension $q$ effective singular meromorphic foliation on $Y$ given by an exact sequence (1). Since $T Y \cong \boldsymbol{O}_{Y}^{\oplus n}$, we have $E \cong \boldsymbol{O}_{Y}^{\oplus q}, F$ is regular and $F$ is induced by a linear foliation of $\mathbf{C}^{n}$. Now assume $n=2$ and $q=1$. Fix any $P \in Y$. Let $\pi: M \rightarrow Y$ be the blowing-up of $Y$ at $P$. The foliation $F$ induces a singular meromorphic foliation, $H$, by curves on $M$. Since $h^{0}\left(Y, T Y \otimes \boldsymbol{I}_{P}\right)=0$, we see that the tangent sheaf $T_{H}$ of the foliation $H$ is isomorphic to $\boldsymbol{O}_{M}\left(-\pi^{-1}(P)\right)$. In particular we have $h^{0}\left(M, T_{H}\right)=0$. Take any smooth compact complex surface $X$ whose minimal model is isomorphic to $Y$ but with $X \neq Y$ and let $\Phi$ be the singular meromorphic foliation on $X$ induced by $F$. Since $X \neq Y$, the minimal model morphism $X \rightarrow Y$ factors through the blowing-up of a point of $Y$. Thus $h^{0}\left(X, T_{\Phi}\right)=0$.

Proof of 1.5. Let $\boldsymbol{O}_{X}(D), D \geq 0$, be the tangent line bundle to the foliation $F$. Let $G$ be a general fiber of $\alpha$. By [U2], Main Theorem I, or [U3], Th. 5.1, $G$ is a smooth connected complex surface with $\kappa(G)=0$. Let $Y$ be the minimal model of $G$. By the generality of $G$ the foliation $F$ induces a non-zero morphism $u: \boldsymbol{O}_{G}(D \mid G) \rightarrow T X \mid G$ which is an inclusion, except at most at finitely many points of $G$. Let $Y$ be the minimal model of $G$. Since $G$ is a smooth fiber of $\alpha$, the normal bundle of $G$ in $X$ is trivial. Hence $T X \mid G$ is an extension of $\boldsymbol{O}_{G}$ by $T G$. By [BH], Th. 4.1, we have $2\left(h^{1}\left(G, \boldsymbol{O}_{G}\right)\right)=b_{1}(G)+\Delta$ and $h_{0}\left(G, \Omega_{G}\right)=h^{1}\left(G, \boldsymbol{O}_{G}\right)-\Delta$ with $0 \leq \Delta \leq 1$. We distinguish two cases. 
(a) Here we assume $D \mid G$ trivial. Notice that this case cannot occur if $F$ is strictly generically effective. In this case $F$ is semi-Riccati. First assume that the composition, $v$, of $u$ with the natural surjection $T X \mid G \rightarrow \boldsymbol{O}_{G}$ is not zero. Since $D \mid G$ is trivial, $v$ is an isomorphism and hence the singularity set of $F$ is disjoint from $G$ and the foliation $F$ is transversal to $G$ at each point of $G$, contradicting one of our assumptions. Now assume $v=0$. Thus $u$ induces a singular meromorphic foliation on $G$ whose tangent bundle is the trivial line bundle. By Remarks 2.4, 2.5 and 2.6 and the assumption $\kappa(G)=0$, we obtain that $Y$ is a torus, that the foliation on $G$ induces by $u$ is induced by a regular foliation, $H$, on $Y$ and that $Y$ is just a foliation induced by a linear foliation of $\mathbf{C}^{2}$. The last part of Remark 2.6 implies $G=Y$. Now assume that $Y$ is a primary Kodaira surface ([BPV], pp. 146-147). Thus $K_{Y} \cong \boldsymbol{O}_{Y}, \Delta=1, b_{1}(Y)=3, h^{1}\left(Y, \boldsymbol{O}_{Y}\right)=2$ and hence $h^{0}(Y, T Y)=h^{0}\left(Y, \Omega_{Y}\right)=1$ and there is a submersion $m: Y \rightarrow B, B$ a smooth elliptic curve, such that all fibers of $m$ are isomorphic to a smooth elliptic curve $C$. Since $h^{0}(Y, T Y)=1$, there is a unique effective singular meromorphic foliation on $Y$ : the foliation is induced by the submersion $m$. Hence the foliation on $G$ induced by $\operatorname{Im}(u)$ is the strict transform of the foliation, $H$, on $Y$ induced by the submersion $m$. The last part of Remark 2.6 implies $G=Y$.

(b) Here we assume $D \mid G \neq \emptyset$. Thus $\operatorname{Im}(u) \subset T G$ and $u$ induces a singular meromorphic foliation on $G$. By Remarks 2.4, 2.5 and $2.6 Y$ is a torus or a primary Kodaira surface. First assume that $Y$ is a torus. We easily obtain as in (a) that the foliation on $G$ induced by $u$ is induced by a linear foliation, $H$, on $Y$. Since the tangent sheaf to the foliation $H$ is trivial, as in case (a) we obtain $G=Y$ and $D \mid G \equiv 0$, contradiction. Now assume that $Y$ is a primary Kodaira surface. As in case (a) we obtain $G=Y$ and $D \mid G \equiv 0$, contradiction.

Proof OF 1.6. Let $E \subset T X$ be the rank $q$ tangent sheaf of the foliation $F$. Let $G^{\prime}$ be a general fiber of $\alpha$ and $G$ any connected component of $G^{\prime}$. Thus $G$ is a smooth compact complex manifold of dimension $m$ and its normal bundle $N_{G / X}$ in $X$ is trivial. Notice that $T X \mid G$ is an extension of $N_{G / X} \cong \boldsymbol{O}_{G}^{\oplus(n-m)}$ by $T G$. Since $G^{\prime}$ is general, the inclusion $E \rightarrow T X$ induces an injection, $u$, of $E \mid G$ into $T X \mid G$. Since $F$ is strictly generically positive, we have $h^{0}\left(G, \operatorname{Hom}\left(E \mid G, \boldsymbol{O}_{G}\right)\right)=0$. Thus $u$ induces an injection $v: E \mid G \rightarrow T G$. Hence $\operatorname{dim}(G) \geq \operatorname{rank}(\operatorname{Im}(v))=q$. If $q=m$, we also obtain that $T G$ is strictly generically positive. Now assume $q<m$. Since $E$ is involutive, $\operatorname{Im}(v)$ is involutive, i.e. $\operatorname{Im}(v)$ defines a dimension $q$ singular meromorphic foliation on $G$.

\section{References}

[BPV] W. Barth, C. Peters and A. VAn De Ven, Compact Complex Surfaces, Ergebnisse der Math. 3. FolgeBand 4, (1984), Springer.

[BH] E. BOMbIERI and D. HuSEMÖLLER, Classification and embeddings of surfaces, Algebraic geometry, Arcata 1974, Amer. Math. Soc. Proc. Symp. Pure Math. 29 (1975), 329-423.

[B] M. BRUnella, Feuilletages holomorphes sur les surfaces complexes compactes, Ann. scient. Ec. Norm. Sup. 30 (1997), 569-594.

[G] X. GomeZ-Mont, Holomorphic foliations in ruled surfaces, Trans. Amer. Math. Soc. 312 (1989), 179201. 
[H] R. Hartshorne, Stable reflexive sheaves, Math. Ann. 254 (1980), 121-176.

[MS] D. Mumford and K. SuOMinen, Introduction to the theory of moduli, Algebraic Geometry, Oslo 1970, Wolters-Noordhoff Publishing, (1972), 171-222.

[OSS] C. Okonek, M. Schneider and H. Spindler, Vector Bundles on Complex Projective Spaces, Progress in Math. 3 (1987), Birkhäuser.

[U1] K. UENo, Classification theory of algebraic varieties and compact complex spaces, Lect. Notes in Math. 439 (1975), Springer.

[U2] K. UENO, On compact analytic threefolds with non-trivial Albanese tori, Math. Ann. 278 (1987), 41-70.

[U3] K. UENo, Degeneration of elliptic surfaces and certain non-Kähler manifolds, Classification of Algebraic and Analytic Manifolds, Progress in Math. 39 (1983), Birkhäuser, 545-566.

\section{Present Address:}

Department of Mathematics, University of Trento,

38050 Povo (TN), ITALY.

e-mail: ballico@science.unitn.it 